\title{
Structure of domains in random Ising magnets
}

\author{
U. Nowak* and K. D. Usadel \\ Theoretische Tieftemperaturphysik, Universität Duisburg, Lotharstrasse 1, 4100 Duisburg 1, Germany
}

(Received 25 February 1992)

\begin{abstract}
We investigate the behavior of domains in random Ising magnets by use of a Monte Carlo simulation. The discovery and investigation of fractality and interpenetration in the structure of the domains of two- and three-dimensional diluted antiferromagnets in an external magnetic field leads to new theoretical considerations concerning the behavior of a domain state. To emphasize the relevance of these considerations for experimental findings we calculate the spatial correlation function of this frozen domain state and compare the results with those line shapes found from neutron-scattering experiments. We give an alternative interpretation of the corresponding correlation length.
\end{abstract}

\section{INTRODUCTION}

One starting point for the theoretical investigation of the behavior of random magnets and related systems is to consider the system as consisting of finite ordered regions, called clusters, domains, or droplets. Several assumptions have to be made for these objects in order to treat them analytically, such as being more or less compact with a typical size, being noninterpenetrating, without domains inside domains, fulfilling scaling relations as usual Euclidean objects.

Although assumptions such as these may be useful for many systems we will show that they are not trivially fulfilled. We demonstrate this for the domains of diluted Ising antiferromagnets in an external magnetic field (DAFF) although many of the following considerations may be valid for other related systems as well.

The domains of the DAFF have qualities completely deviating from the assumptions mentioned above, since they are fractal and interpenetrating, forming a hierarchy of domains inside domains on all length scales which are observable. In order to underline the relevance of these findings we compare the correlation function of the domain state of the DAFF, determined through a Monte Carlo simulation, with the corresponding line shapes of neutron scattering. Our results are in good agreement with the experimental ones. An investigation of the structure of the occurring domains leads to a new interpretation of the corresponding correlation length differing from the usual interpretation.

The organization of the paper is as follows.

In the next section we will report on the model we choose for our investigation and explain our simulation techniques.

In the third section we focus on the structural properties of the frozen domain state of the DAFF. We show the domains to be fractal in both two and three dimensions and discuss their structure and corresponding scaling relations.

In Sec. IV we compare our simulation results with the experimental ones and illustrate the importance of our considerations for the interpretation of neutronscattering results.
Finally, we give a brief summary in the last section.

\section{THE MODEL}

The Hamiltonian of the DAFF in units of the coupling constant $J$ reads

$$
H=\sum_{\langle i, j\rangle} \epsilon_{i} \epsilon_{j} \sigma_{i} \sigma_{j}-B \sum_{i} \epsilon_{i} \sigma_{i}
$$

where $\sigma_{i}= \pm 1$ and $\epsilon_{i}=0,1$.

This system is in the same universality class as the random-field Ising model (RFIM) ${ }^{1}$ Its phase diagram is still not completely understood (see Refs. 2 and 3 and references therein). A well-known feature of the DAFF is the formation of a domain state with extremely long relaxation times. This domain state is thought to be metastable for lower fields and it is obtained by cooling the system in an external field from the paramagnetic high temperature phase. From neutron-scattering experiments it was concluded that this field-cooled state is frozen at low temperatures. ${ }^{4}$ The mechanisms which are responsible for the corresponding hysteretic properties of the DAFF have been investigated experimentally, ${ }^{4,5}$ theoretically, ${ }^{6}$ and in computer simulations. ${ }^{7,8}$ Apart from this a nonexponential decay of the remanent magnetization of the field-cooled state after switching off the external field has been found experimentally ${ }^{5}$ and further investigated theoretically by a scaling theory ${ }^{9}$ and in computer simulations, respectively. ${ }^{10}$ Here we want to focus on the structural properties of the frozen domains in both two- and three-dimensional systems.

In our Monte Carlo simulation ${ }^{11}$ we considered nearest-neighbor interaction only. We used helical boundary conditions and the heat-bath algorithm. Typical sizes of the simple cubic, respectively, square lattices were $99 \times 99 \times 98$ and $199 \times 198$ but even larger lattices with sizes of up to $145 \times 145 \times 144$ and $999 \times 998$ have been investigated in order to confirm our findings on larger length scales. The dilution $p$ was $50 \%$ (3D) and $25 \%$ (2D).

All programs have been written in $\mathrm{C}$ and the simulation was done on IBM/RS6000 work stations. 
The formation of a domain state can be reached by cooling the system in an external magnetic field $B$. In the simulation we use a completely random distribution of spins as the initial configuration, corresponding to a paramagnetic state. Then we cool the system in the field by lowering the temperature step by step. A domain state develops, the structure of which depends on the cooling rate, i.e., on the number of Monte Carlo steps per spin (MCS) per temperature step. By cooling the system slowly enough a practically frozen domain state can be reached. This domain state is metastable in the sense that there is no observable development toward antiferromagnetic long-range order.

In the next section we will analyze the structure of this domain state.

\section{STRUCTURAL QUANTITIES}

The geometrical properties of objects such as domains, droplets, or clusters in random magnets or equivalent systems are important as a starting point for theoretical considerations as well as for the interpretation of experimental results (see, e.g., the cluster theory of spin glasses, ${ }^{12}$ the droplet theory for thermal fluctuations, ${ }^{13}$ or the domain theory of random-field systems ${ }^{6}$ ).

All these theories are based on the assumption that the system consists of domains of ordered spins. These domains are thought to be compact, noninterpenetrating, and without a hierarchy of domains inside domains. They fulfill Euclidean scaling relations between quantities such as radius and volume and only a surface which is fractal on small length scales sometimes is admitted. ${ }^{9}$

Figure 1 shows a typical two-dimensional domain state of the DAFF. The black and grey regions correspond to the two possible antiferromagnetic phases. Vacancies are not shown. This domain state has been developed by cooling a $399 \times 398$ system for a time of 55000 MCS in

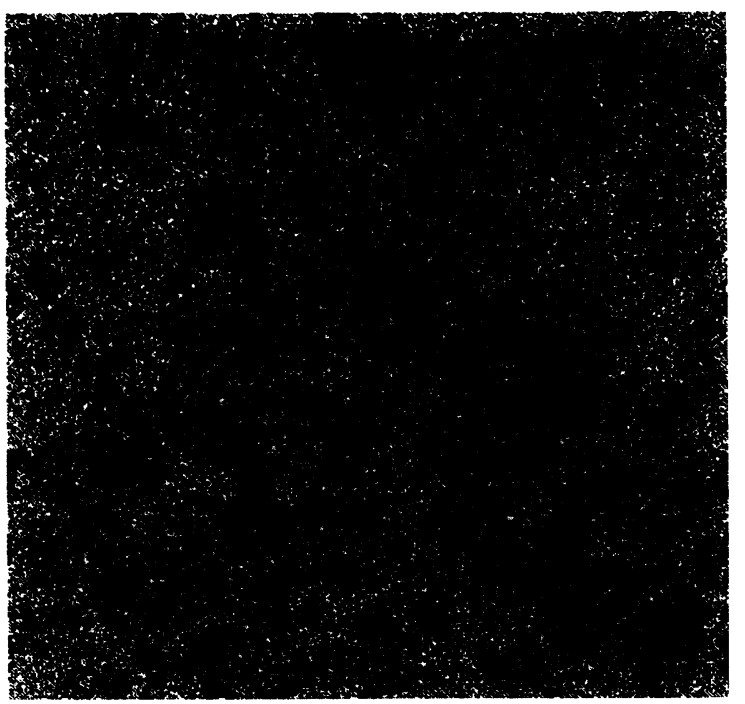

FIG. 1. Frozen domain state of a $399 \times 398$ system. The two phases are represented in black and grey. Vacancies are left white.

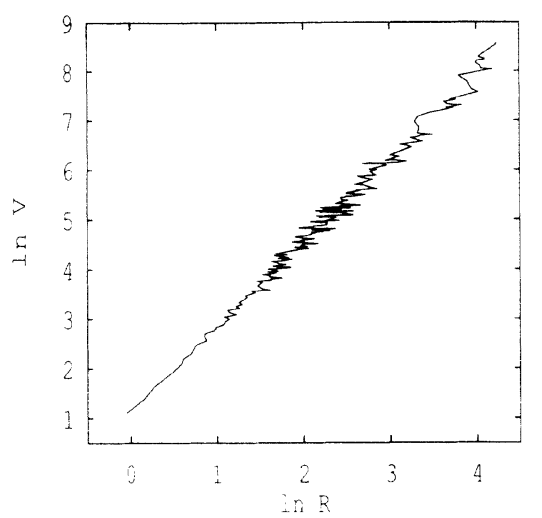

FIG. 2. $\ln V$ vs $\ln R$. All quantities are dimensionless and explained in the text.

a field of $B=1.5$. The temperature at the end of the procedure is $T=0.9$.

In order to investigate the structure of the domain state we perform a cluster analysis with a suitable adjusted Hoshen-Kopelman-type algorithm. ${ }^{14}$ This algorithm pieces the system into domains of connected and antiferromagnetically ordered spins. In this way it is possible to compute directly the volume $V$ (number of spins), surface $F$ (number of unsatisfied bonds), and radius $R$ (root of the mean-squared distance of spins) of the domains formed (note that in two dimensions $V$ is the area and $F$ the border of the domains).

In order to get a sufficient large number of domains we repeated the procedure described above for many lattices (typically 50 systems of a size of $199 \times 198$ ). Then, all quantities were averaged over domains of equal volume. Figure 2 shows the resulting data of $\ln V$ versus $\ln R$ and Fig. $3 \ln V$ versus $\ln F$. The data suggest the following scaling relations:

$$
V \sim R^{D}
$$

and

$$
V \sim F^{D^{\prime}} \text {. }
$$

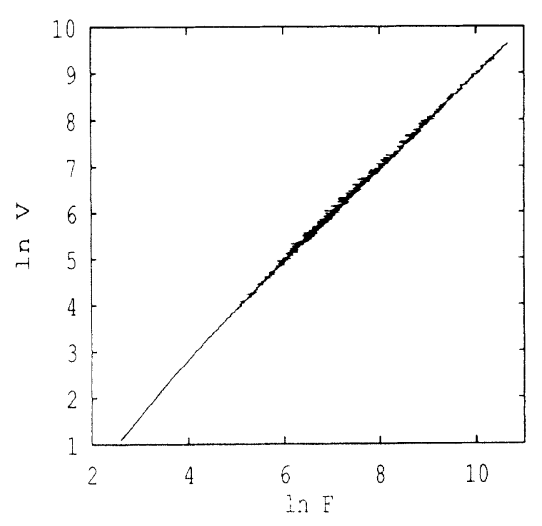

FIG. 3. $\ln V$ vs $\ln F$, corresponding to Fig. 2. 
Fitting the data to Eqs. (2) and (3) we get the exponents $D=1.56 \pm 0.03$ and $D^{\prime}=1.001 \pm 0.002$. The fact that $V \sim F$ is not unusual for highly diluted objects since most of the surface is inside the domains. Note that inner surfaces result from the dilution as well as from a hierarchy of domains inside domains. This is obvious in Fig. 1 and additionally it will be proven below. The fractal dimension $D=1.56$ from the volume-radius relation is known from the so-called lattice animals of the percolation problem. ${ }^{15}$ Our conclusion is that the magnetic domains of the DAFF show the same fractal behavior as the-in our system much smaller-finite clusters of the percolation problem. The infinite cluster of sites occupied with magnetic ions splits into finite domains which have the same fractal dimension as the finite clusters of the percolation problem. The analysis of the domain state of the three-dimensional DAFF has been reported earlier (see Sec. IV in Ref. 3) and corresponding results have been obtained , i.e., it is $V \sim F$ and $V \sim R^{D}$ where $D=2$ is the fractal dimension of the three-dimensional lattice animals. ${ }^{16}$

It should be mentioned that in the simulations normally very large domains can occur exceeding the size of the system. In three dimensions, a large number of spins may belong to two percolating domains of the two different phases. This may be a finite-size effect vanishing for larger lattices. However, in three dimensions there is the possibility of a domain state containing two infinite domains interpenetrating each other. This does not mean, of course, that the system is long-range ordered in the usual sense. The order parameter remains zero if the two infinite domains belong to the two different phases. Note that it has been pointed out already earlier, that in the description of phase transitions as a percolation transition of ordered clusters these clusters differ from the domains here. ${ }^{17}$

Now, we want to focus on the relevance of our findings with respect to the scaling of physical properties. The first estimate of the influence of random fields on $d$-dimensional spin systems came from Imry and Ma. ${ }^{18}$ Assuming the domains of the DAFF to be compact with a typical radius $R$ we will find a statistical surplus of vacancies in one of the antiferromagnetic sublattices of the domain which scales with $R^{d / 2}$. This leads to a net magnetization which couples to the homogeneous external field decreasing the energy of the system by

$$
E_{m} \sim-B R^{d / 2} .
$$

This fact explains the existence of finite domains. On the other hand there is an increase of energy through the broken bonds in the domain wall:

$$
E_{w} \sim R^{d-1}
$$

Comparing these two scaling relations one finds that depending on the dimensionality $d$ in an external magnetic field it may be favorable to create domains, i.e., longrange order is lost.

Even though this argument is rather simple it is impressive as a first-principle ansatz and much of the newer

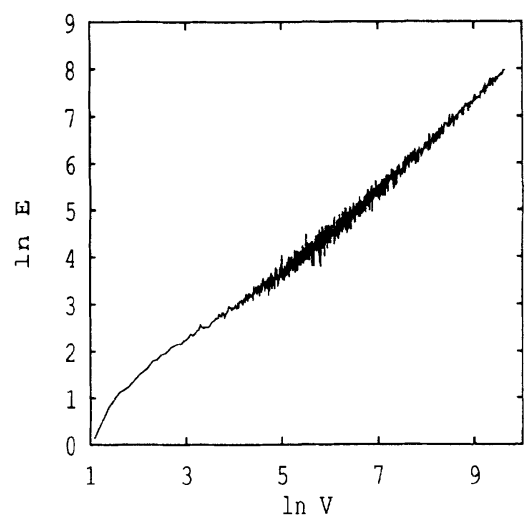

FIG. 4. $\ln E_{W}$ vs $\ln V$, corresponding to Fig. 2 .

theoretical work is based on similar assumptions. ${ }^{6,9}$

The validity of the relations above can be deduced directly by a simulation.

After having split the frozen domain state into the finite domains we can compute the corresponding quantities volume magnetization $M_{V}$ and domain-wall energy $E_{W}$ ( number of broken bonds) for all domains. Figures 4 and 5 show $E_{W}$ and $M_{V}$ versus the volume $V$ of the domains for the two-dimensional system, computed in the same way as Figs. 3 and 2. The following scaling relations hold for $V$ large enough:

$$
E_{W} \sim V^{0.98 \pm 0.01}
$$

and

$$
M_{V} \sim V^{1.00 \pm 0.01} \text {. }
$$

These relations clearly indicate deviations from the Imry-Ma-type assumptions made above. Similar deviations have been reported earlier for the three-dimensional $\mathrm{DAFF}^{3}$ and for the RFIM. ${ }^{19}$

Figure 6(a) illustrates the usual understanding of a domain state (note that often in the literature not a domain state is considered but only single domains in a longrange ordered background). The domains are compact

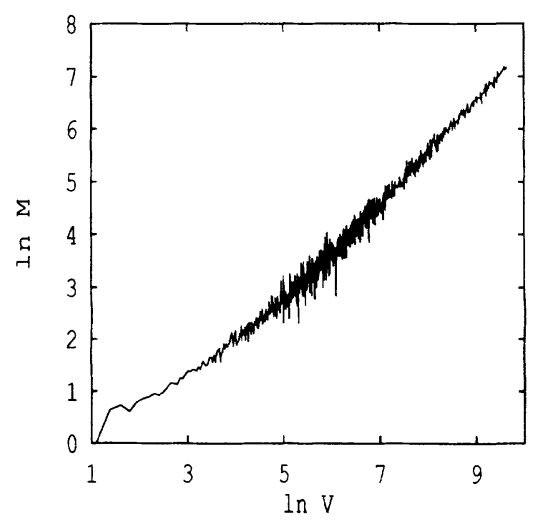

FIG. 5. $\ln M_{V}$ vs $\ln V$, corresponding to Fig. 2. 
and well defined through a typical radius. In contrast to this picture Fig. 6(b) shows a more realistic domain state according to the simulation result Fig. 1 . These domains have inner surfaces which in the case of the DAFF on the one hand may result from vacancies explaining the fact that the surface is proportional to the volume. On the other hand even inner domains are obviously relevant since even the domain-wall energy is proportional to the volume of the domains. Another important feature of these domains is the structure. These domains are not compact so that a statistical square-root argument [Eq. (4)] does not hold. Instead, they have a complex structure taking advantage from random-field fluctuations on all length scales. This leads to the fractality of the domains itself, not only to a fractality or roughness of their domain wall. ${ }^{9}$

Comparing our results with earlier numeric studies we note that in Ref. 19 the authors could not distinguish whether the volume or the surface of the domains fulfill fractal scaling relations since they determined the fraction of the corresponding exponents only. For the twodimensional RFIM the domains have been found to be fractal, but with the fractal dimension of the percolating cluster at the critical concentration $\left(d_{f}=\frac{91}{48}\right) .{ }^{20}$

In the next section we want to show that our findings are relevant for the understanding of experimental results.

(a)

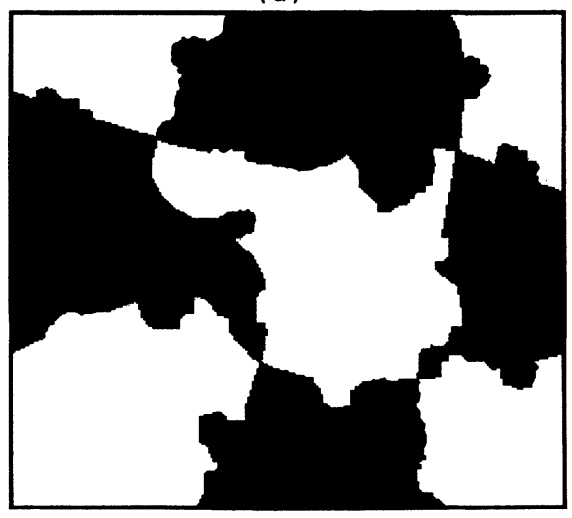

(b)

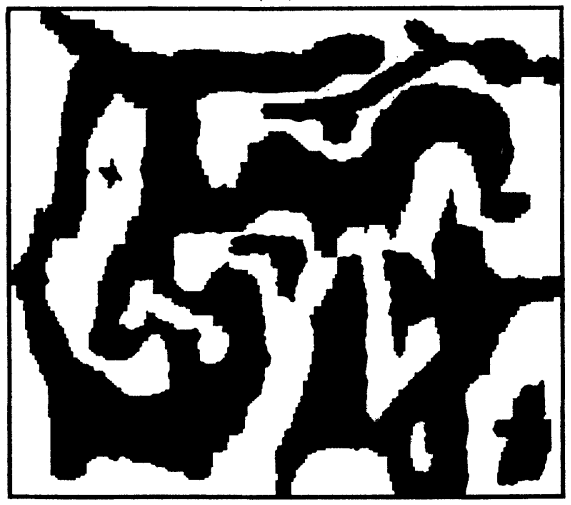

FIG. 6. Scheme of a domain state illustrating (a) the usual picture of the Imry-Ma argument and (b) the more realistic behavior found in simulations.

\section{SPATIAL CORRELATIONS}

Neutron scattering is a well-established method to investigate the structural properties of magnetic systems experimentally. From neutron-scattering experiments it was concluded that the domain state of the DAFF is frozen at low temperatures ${ }^{4}$ since it has a finitecorrelation length being constant in time. Performing quasielastic scattering one measures the scattering line shape $I(q)$ which is the Fourier transform of the spinspin correlation function $c(\mathbf{r})=\left[\left\langle\sigma\left(\mathbf{r}_{0}\right) \sigma\left(\mathbf{r}_{0}+\mathbf{r}\right)\right\rangle\right]$, where $\langle\cdots\rangle$ denotes a thermal average and $[\cdots]$ a configurational average.

Within the Ornstein-Zernike approach the correlation function in the critical region of the three-dimensional Ising systems has the form

$$
c(r) \sim r^{-1} e^{-r / \xi}
$$

leading to the well-known Lorentzian form of the scattering line shape:

$$
I(\mathbf{q}) \sim \frac{1}{\kappa^{2}+q^{2}},
$$

where $\xi$ is the correlation length and $\kappa=1 / \xi$. Although the exact form of the scattering line shape in the case of the frozen domain state of the DAFF is not well understood it is a well-established procedure to fit the scattering data predominantly to a squared Lorentzian for threedimensional systems perhaps with a small Lorentzian correction term. ${ }^{21,22}$ Theoretical arguments for this kind of line shape have been given. ${ }^{23}$

In two dimensions the data are either fitted to a squared Lorentzian as well or to a Lorentzian to the power of $1.5 .^{24}$

It is interesting to note that the squared Lorentzian and the Lorentzian to the power of 1.5 are the Fourier transform of pure exponentially decaying correlation functions in three and two dimensions, respectively. Since the exponential decay of the correlation function usually has power-law corrections as in Eq. (8) it is useful to look for power-law corrections of exponentially decaying correlation functions.

We used the Monte Carlo simulation to determine the correlation function $c(r)$ directly. In a two-dimensional system of size $L \times(L-1)$ the staggered correlation function $c(r)$ is determined as

$c(r)=\left[\left\langle\frac{1}{2 L} \sum_{i=1}^{L}(-1)^{r}\left(\sigma_{i, \frac{L}{2}} \sigma_{i, \frac{L}{2}+r}+\sigma_{i, \frac{L}{2}} \sigma_{i, \frac{L}{2}-r}\right)\right\rangle\right]$.

In three dimensions the correlation function is determined correspondingly as the average correlation of spins with perpendicular distance $r$ from the mean plane of the system with the spins in this plane. Periodic as well as free boundary conditions have been employed in this direction in order to investigate the influence of the surface of the finite lattice. We found that confining the determination of $c(r)$ to small enough $l$, i.e., $l<L / 2$ we can 
neglect the influence of free boundary conditions.

Figure 7 shows a semilogarithmic plot of $c(r)$ for three (a) and two (b) dimensions during field cooling. In three dimensions 25 systems of linear sizes ranging from $L=99$ to 49 have been cooled in a field of $B=0.5$ in temperature steps $\Delta T=0.1$ from a temperature $T_{\max }=1.9$ (lowest curve) to $T_{\min }=0.8$ (topmost curve) with a cooling rate of $1000 \mathrm{MCS} / \Delta T$.

The field cooling of the two-dimensional systems has been performed for 50 systems with $L$ ranging from 199 to 99 , a field of $B=0.5$, a cooling rate of $5000 \mathrm{MCS} / \Delta T$ and for temperatures ranging from 1.7 (lowest curve) to 0.8 (topmost curve) with $\Delta T=0.1$.

In both figures a freezing of the system can be observed since the correlation functions are nearly parallel for lower temperatures. This means that the correlation length which is more or less the slope of the curves remains constant in agreement with the experimental findings. A straight line in the semilogarithmic plot of Fig. 7 gives evidence for an exponential decay of $c(r)$. Therefore we fit the low temperature data of the frozen domains state to the general form

$$
c(r)=c_{0} r^{-x} e^{-r / \xi} \text {. }
$$

Neglecting the first two points of the $c(r)$ curves we get exponents $x$ being less than 0.1 . These very small power-
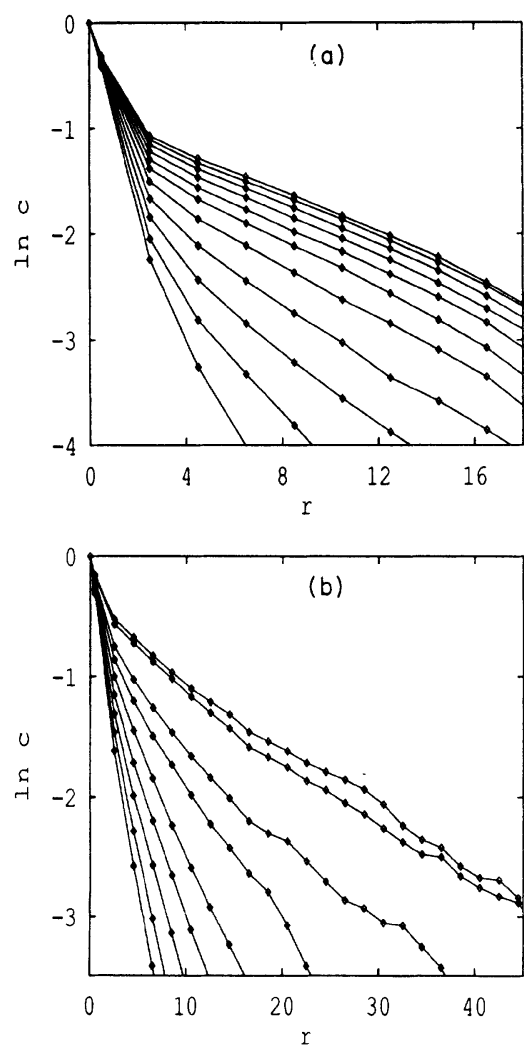

FIG. 7. $\ln c$ as defined in the text vs $r$ for different temperatures during field cooling (from below). (a) is for a threedimensional system and (b) the corresponding figure for two dimensions. law corrections give evidence for the fact that $c(r)$ is well described by a pure exponential for the frozen domain state of the DAFF confirming the scattering line shapes $I(q)$ being well fitted by a squared Lorentzian (3D) and a Lorentzian to $1.5(2 \mathrm{D})$, respectively.

Now we want to focus on the dependence of the correlation length $\xi$ on the cooling rate and the external field. In the following we neglect the small power-law corrections and we determine $\xi$ by directly fitting to a pure exponential. Note that we always confirmed the exponent $x$ to be very small by fitting the data to Eq. (11) as well.

Figure 8 shows a semilogarithmic plot of the low temperature data of $c(r)$ after field cooling for different external fields. As earlier, Fig. 8(a) corresponds to the three-dimensional system and (b) to the two-dimensional system. The resulting correlation lengths are shown in Fig. 9. Field cooling has been performed with different cooling rates in order to investigate the corresponding dependence of $\xi$ on the cooling rate. The different curves belong to different cooling rates (in Fig. 8 the data resulting from the slowest cooling are shown). In the two-dimensional case [Fig. 9(b)] it is obvious that a simple power law holds for slow enough cooling rates. Fitting yields $\xi \sim B^{-1.52 \pm 0.04}$ in agreement with the experimental finding $\xi \sim B^{-1.6} .{ }^{24}$ In three dimensions the dependence of $\xi$ on the cooling rate is stronger and more difficult to overcome. However, in the limit of high fields
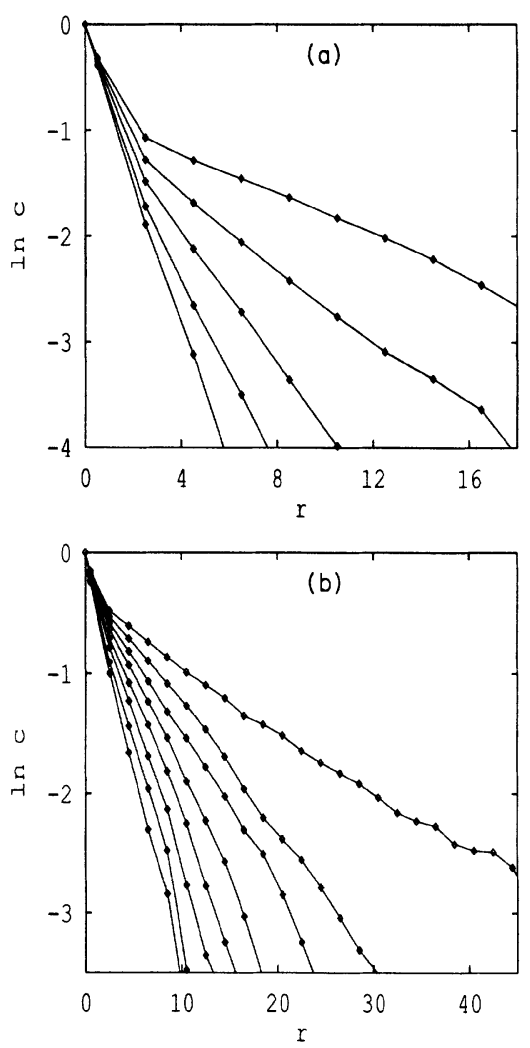

FIG. 8. $\ln c$ vs $r$ after field cooling in different fields. (a) (3D) $B=0.5,0.9,1.3,1.7,2.1$ (from above); (b) (2D) $B=$ $0.5,0.7,0.9,1.1,1.3,1.5,1.7,1.9$ (from above). 
[the last two points in Fig. 9(b)] there are no time dependences observable. The corresponding power-law relation is $\xi \sim B^{-2.3}$. This is in agreement with the experimental findings $\xi \sim B^{-2.2 \pm 0.1}$ (see Ref. 22 and references therein).

The simulations with slow cooling rates are extremely time consuming and we would need even longer simulations to confirm the power law above for lower fields. Additionally, a finite-size analysis would be necessary if the correlation length exceeds the lattice size. Since it is not our intention to question the scaling behavior above for lower fields we leave out this part of the analysis.

Instead, we would like to compare the correlation lengths observed with the ones determined in experiments. The correlation lengths in our simulations range from about 3 to about 18 . The correlation lengths determined through neutron scattering for the DAFF range from 240 to about 1 (Refs. 25 and 26) (all lengths cited here are measured in units of the lattice constant). Surprisingly, the experimental results in the limit of high fields and high dilution and our results are of the same order of magnitude even though experimental systems are much larger, of course, than ours. This, together with the fact that the scaling of $\xi$ with $B$ coincides with experimental results shows that the structural findings of our simulations are relevant for the length scales in experimental systems as well.
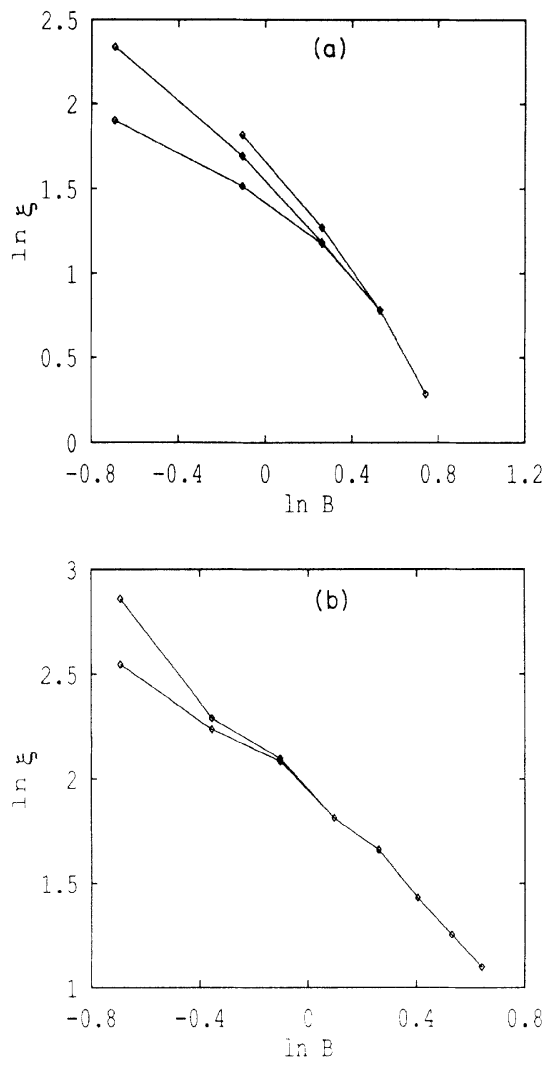

FIG. 9. $\ln \xi$ vs $\ln B$ for different cooling rates. (a) (3D) $1000,500,300 \mathrm{MCS} / \Delta T$ (from above); (b) (2D) 5000,1000 $\mathrm{MCS} / \Delta T$ (from above)
Usually domains are thought to be much larger in real materials. Therefore we want to focus now on the question of how to interpret the correlation length. Figure 1 shows the domain state arising after field cooling in a field of $B=1.5$ with a cooling rate of $5000 \mathrm{MCS} / \Delta T$. The corresponding correlation length [see Fig. $9(\mathrm{~b})$ ] is about 3.5. Since Fig. 1 represents a $399 \times 398$ system this is less than $1 \%$ of the linear lattice size and therefore certainly not the radius of the largest domain which has a volume of 22396 spins corresponding to a radius of about 148 . Also, $\xi$ is not a mean or typical radius. Due to the fractality of the domains the structure is selfsimilar on all occurring length scales. Therefore, there is no typical length scale in the system. Figure 10 shows a double-logarithmic plot of the distribution of domains of a given volume, i.e., the logarithm of the number of domains versus the logarithm of its volume. There is a broad distribution of domains with no peak or even maximum which would give rise to a typical, i.e., frequent domain size. Instead, Fig. 10 suggests that the distribution follows a power law in agreement to what is known for other fractal systems, e.g., in the percolation problem. ${ }^{15}$

Nevertheless the definition of a mean-domain size is possible even though this size is not the most one. The mean size of the domains in the system shown in Fig. 1 is $V=762$ corresponding to a radius of about 26 . Even this quantity is much larger than the correlation length.

From the considerations above we conclude that $\xi$ is a length scale which has nothing to do with the size of domains but can be interpreted as being related to the interpenetration of the domains. Figure 6 illustrates this situation. Walking through the system in Fig. 6(b) from one side to the other one changes the phase frequently even though there are large domains. This is possible due to the interpenetration of the domains. The length scale on which domains do interpenetrate does not at all depend on the size of the domains. This is not the case for compact domains as Fig. 6(a) illustrates. Note that in three dimensions there is even the above-mentioned possibility of a system consisting of two percolating domains which interpenetrate each other. A system as this has no divergence in the correlation length seen in neutron-

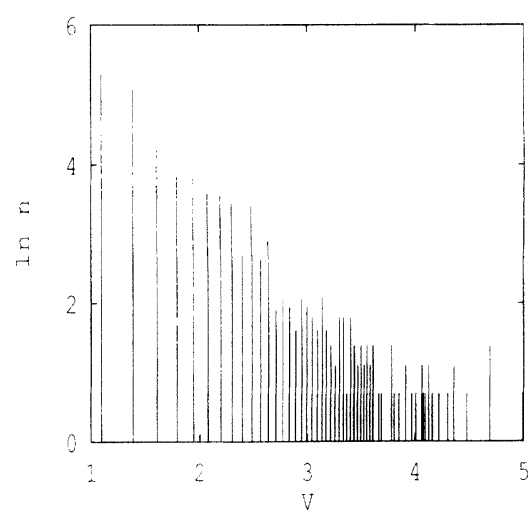

FIG. 10. Double-logarithmic plot of the number of domains $\ln N$ vs the corresponding volume of the domains $\ln V$. 
scattering experiments since this correlation length is obviously related to the interpenetration and not to the domain size.

\section{SUMMARY}

We used the DAFF as a prototype for a system with strong disorder and investigated the behavior of frozen domains by a Monte Carlo simulation. We considered both two- and three-dimensional systems. In both of them we discovered a fractal and interpenetrating structure of the domains.

We further elucidated the relevance of these findings for both, the theoretical understanding as well as the interpretation of experimental results. We discussed the usual assumptions of a droplet-theory ansatz for the un- derstanding of the behavior of domains and its deviations from the results of our simulations. Also, we elucidated the relevance of our findings for the experimental interpretation by simulating the spatial correlation function of a frozen domain state and comparing it with the line shapes found in neutron-scattering experiments. The corresponding correlation length is interpreted in a new way, namely, as a "interpenetration length" rather than a "typical domain size." All these findings can be thought to be relevant for other strongly disordered systems as well, especially for systems which possibly form fractal structures such as spin glasses.

\section{ACKNOWLEDGMENT}

This work was supported by the Deutsche Forschungsgemeinschaft through Sonderforschungsbereich 166.
${ }^{*}$ Electronic address: uli@hal9000.uni-duisburg.de.

${ }^{1}$ S. Fishman and A. Aharony, J. Phys. C 12, L729 (1979).

${ }^{2}$ F. C. Montenegro, A. R. King, V. Jaccarino, S-J. Han, and D. P. Belanger, Phys. Rev. B 44, 2155 (1991).

${ }^{3}$ U. Nowak and K. D. Usadel, Phys. Rev. B 44, 7426 (1991).

${ }^{4}$ R. J. Birgeneau, R. A. Cowley, G. Shirane, and H. Yoshizawa, J. Stat. Phys. 34, 817 (1984); D. P. Belanger, S. M. Rezende, A. R. King, and V. Jaccarino, J. Appl. Phys. 57, 3294 (1985).

${ }^{5}$ U. A. Leitão, W. Kleemann, and I. B. Ferreira, Phys. Rev. B 38, 4765 (1988); P. Pollak, W. Kleemann, and D. P. Belanger, ibid. 38, 4773 (1988).

${ }^{6}$ J. Villain, Phys. Rev. Lett. 52, 1543 (1984).

${ }^{7}$ G. S. Grest, C. M. Soukoulis, and K. Levin, Phys. Rev. B 33, 7659 (1986).

${ }^{8}$ U. Nowak and K. D. Usadel, Phys. Rev. B 39, 2516 (1989).

${ }^{9}$ T. Nattermann and I. Vilfan, Phys. Rev. Lett. 61, 223 (1988).

${ }^{10}$ U. Nowak and K. D. Usadel, Phys. Rev. B 43, 851 (1991); Physica B 165, 211 (1990).

${ }^{11} \mathrm{~K}$. Binder and D. W. Heermann, Monte Carlo Simulations in Statistical Physics (Springer-Verlag, Berlin, 1988).

${ }^{12}$ D. S. Fisher and D. A. Huse, Phys. Rev. Lett. 56, 1601
(1986).

${ }^{13}$ D. A. Huse and D. S. Fisher, Phys. Rev. B 35, 6841 (1987).

${ }^{14} \mathrm{~J}$. Hoshen and R. Kopelman, Phys. Rev. B 14, 3428 (1976).

${ }^{15} \mathrm{D}$. Stauffer, Introduction to Percolation Theory (Taylor and Francis, London, 1985).

${ }^{16}$ G. Parisi and N. Sourlas, Phys. Rev. Lett. 46, 871 (1981).

${ }^{17}$ K. Binder, Ann. Phys. (N.Y.) 98, 390 (1976).

${ }^{18}$ Y. Imry and S. Ma, Phys. Rev. Lett. 35, 1399 (1975).

${ }^{19}$ J. A. Cambier and M. Nauenberg, Phys. Rev. B 34, 7998 (1986).

${ }^{20}$ J. Hong and M. O. Robbins, Phys. Rev. A 44, 2538 (1991).

${ }^{21}$ D. P. Belanger, A. R. King, and V. Jaccarino, Phys. Rev. B 31, 4538 (1985).

${ }^{22}$ H. Yoshizawa, R. A. Cowley, G. Shirane, and R. Birgeneau, Phys. Rev. B 31, 4548 (1985).

${ }^{23}$ I. Vilfan and R. A. Cowley, J. Phys. C 18, 5055 (1985).

${ }^{24}$ H. Yoshizawa, R. A. Cowley, G. Shirane, R. J. Birgeneau, H. J. Guggenheim, and H. Ikeda, Phys. Rev. Lett. 48, 438 (1982).

${ }^{25}$ R. A. Cowley, H. Yoshizawa, G. Shirane, and R. J. Birgeneau, Z. Phys. B 58, 15 (1984).

${ }^{26}$ D. P. Belanger (private communication). 


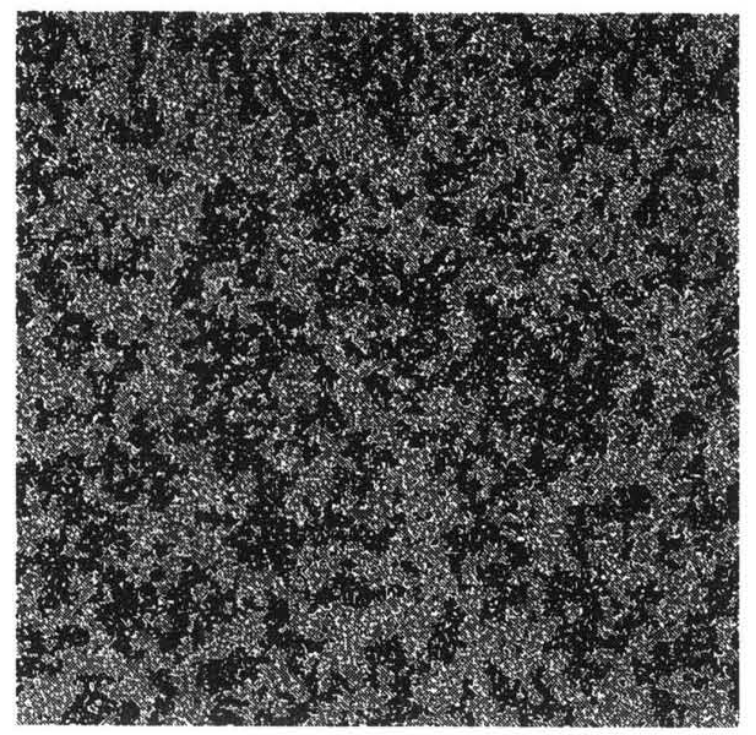

FIG. 1. Frozen domain state of a $399 \times 398$ system. The two phases are represented in black and grey. Vacancies are left white. 